\title{
A novel model to predict cancer-specific survival in patients with early-stage uterine papillary serous carcinoma (UPSC)
}

\author{
Lihua Chen ${ }^{1,2}$, Xi Cheng 1,2 \\ 1 Department of gynecology oncology, Fudan University Shanghai Cancer Center; \\ 2Department of oncology, Shanghai Medical College, Fudan University, Shanghai \\ 200032, China
}

Objective: Stage I-II uterine papillary serous carcinoma (UPSC) has aggressively biological behaviors and leads to poor prognosis. However, clinicopathologic risk factors to predict survival of patients with early-stage UPSC are still unclear. This study was undertaken to develop a prediction model of survival in patients with early-stage UPSC.

Methods: Using SEER database, we identified 964 patients with stage I-II UPSC patients who underwent at least hysterectomy between 20042015. By considering of competing risk events for survival outcomes, we used proportional subdistribution hazards regression to compare cancer-specific death (CSD) for all patients. Based on the results of univariate and multivariate analysis, the variables were selected to construct a predictive model; and the prediction results of the model were visualized by nomogram to predict the cancerspecific survival and the response of adjuvant chemotherapy and radiotherapy.

Results: The median age of the cohort is 67 years old. $17.1 \%(\mathrm{n}=165)$ of the patients died of UPSC (CSD), while $8.6 \%$ of the patients died from other causes (non-CSD). On multivariate analysis, age, tumor size and number of regional nodes removed were significantly associated with cumulative incidence of CSD. In the age $\geq 67$ years cohort, stage I-II was a risk factor for CSD, and the number of lymph node resections greater than 10 was a protective factor. Both combination of adjuvant chemotherapy and radiotherapy and adjuvant chemotherapy alone could decrease CSD of patients with stage I-II UPSC older than 67 years. The prediction model had great risk stratification ability as high risk group had higher cumulative incidence of CSD than lowrisk group. In the high risk group, patients with postoperative adjuvant chemoradiotherapy had improved CSD compared with patients who did not receive radiotherapy nor chemotherapy.

Conclusion: Our prediction model had a good performance in predicting the cancer-specific survival of early-stage UPSC patients and contributed to guide clinical treatment decision.

A

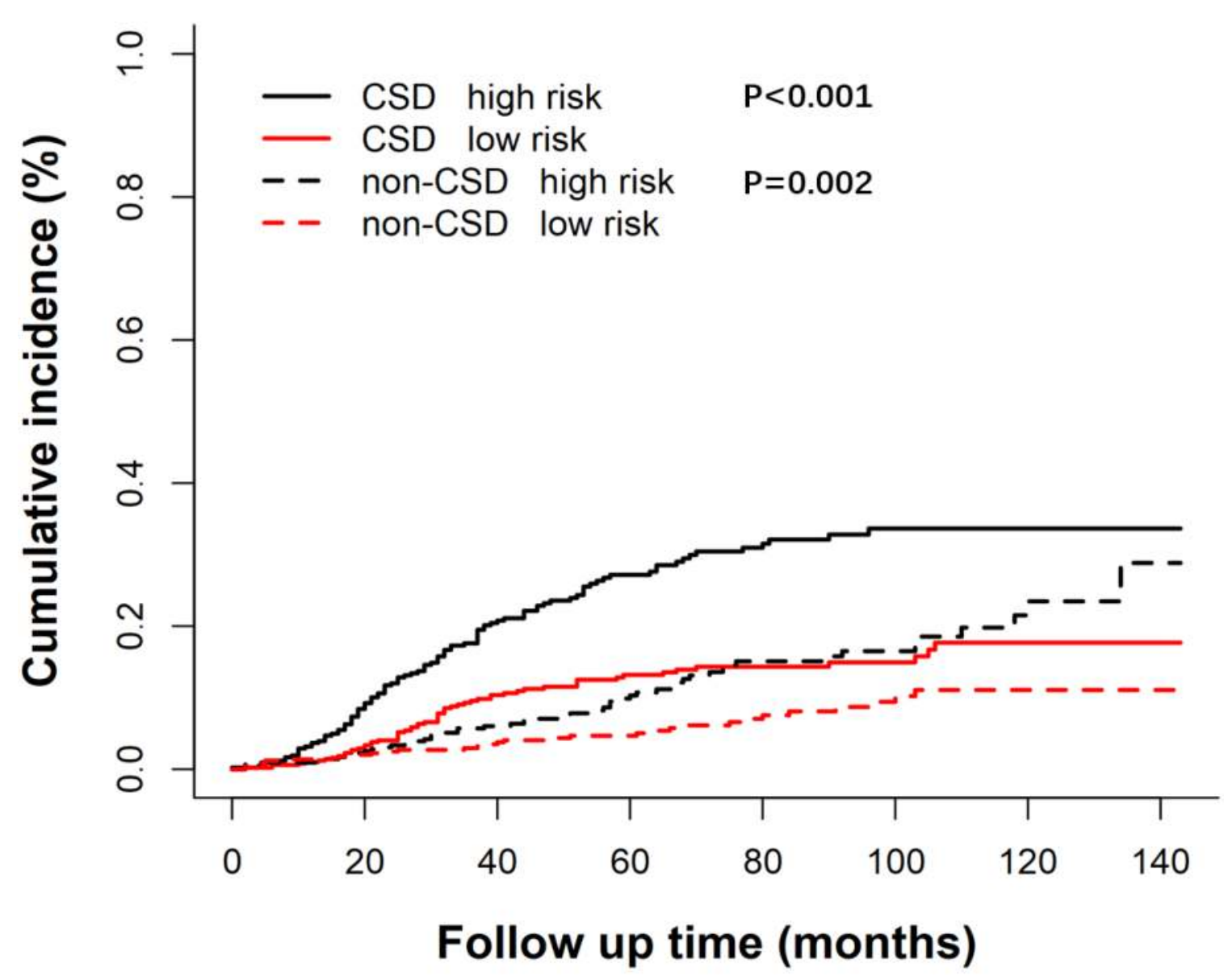

B

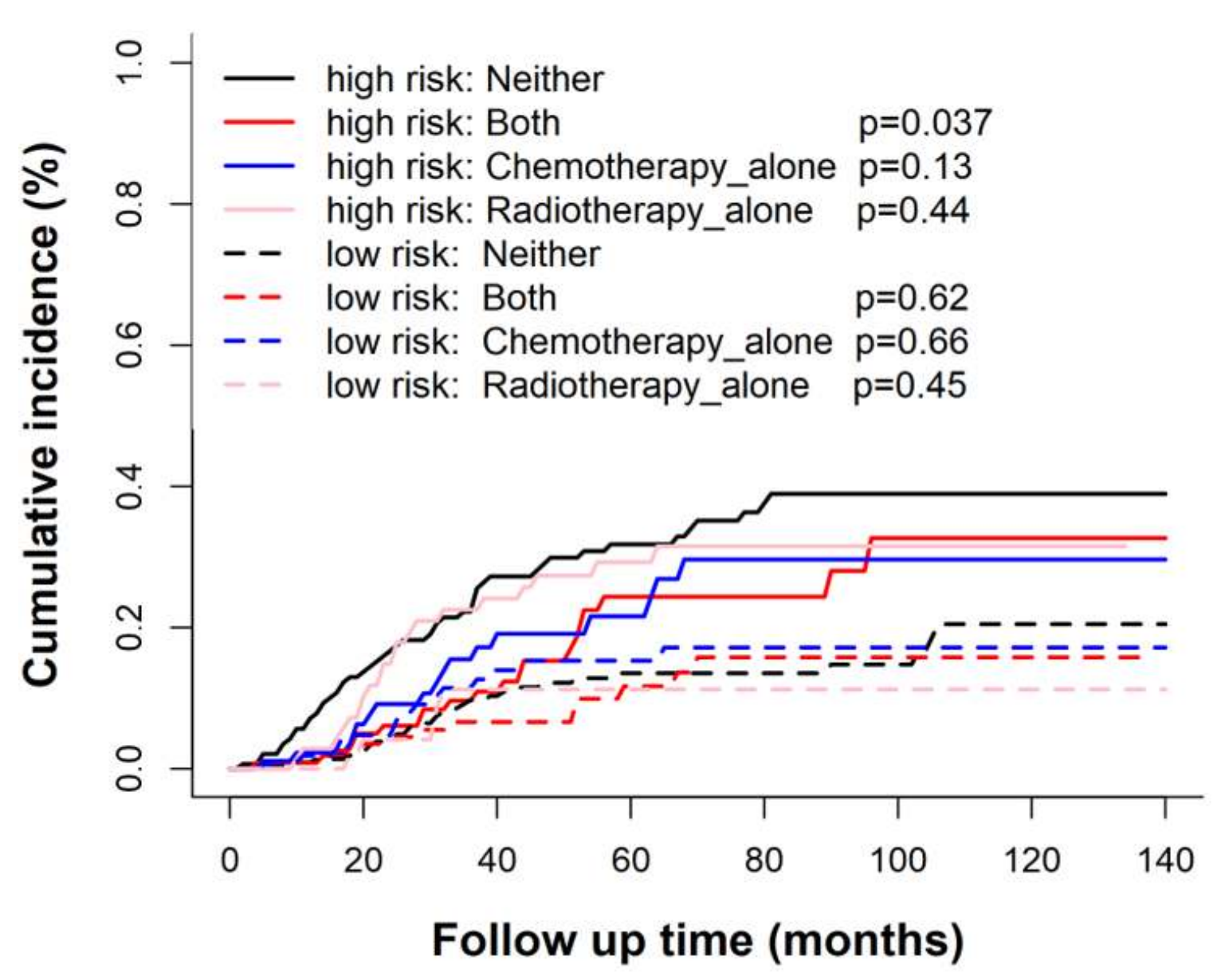

